Article

\title{
Analysis of Optical Solitons for Nonlinear Schrödinger Equation with Detuning Term by Iterative Transform Method
}

\author{
Nehad Ali Shah ${ }^{1,2}{ }^{\mathbb{D}}$, Praveen Agarwal $^{3}\left(\mathbb{D}\right.$, Jae Dong Chung ${ }^{4}\left(\mathbb{D}\right.$, Essam R. El-Zahar ${ }^{5,6 *(\mathbb{D})}$ and \\ Y. S. Hamed 7,8 (D) \\ 1 Informetrics Research Group, Ton Duc Thang University, Ho Chi Minh City 58307, Vietnam \\ 2 Faculty of Mathematics \& Statistics, Ton Duc Thang University, Ho Chi Minh City 58307, Vietnam; \\ nehad.ali.shah@tdtu.edu.vn \\ 3 Department of Mathematics, Anand International College of Engineering, Jaipur 302003, India; \\ goyal.praveen2011@gmail.com \\ 4 Department of Mechanical Engineering, Sejong University, Seoul 05006, Korea; jdchung@sejong.ac.kr \\ 5 Department of Mathematics, College of Science and Humanities in Al-Kharj, \\ Prince Sattam bin Abdulaziz University, P.O. Box 83, Al-Kharj 11942, Saudi Arabia \\ 6 Department of Basic Engineering Science, Faculty of Engineering, Menoufia University, \\ Shebin El-Kom 32511, Egypt \\ 7 Department of Mathematics and Statistics, College of Science, Taif University, P. O. Box 11099, \\ Taif 21944, Saudi Arabia; yasersalah@tu.edu.sa or eng_yaser_salah@yahoo.com \\ 8 Department of Physics and Engineering Mathematics, Faculty of Electronic Engineering, \\ Menoufia University, Menouf 32952, Egypt \\ * Correspondence: er.elzahar@psau.edu.sa
}

Received: 17 October 2020; Accepted: 6 November 2020; Published: 10 November 2020

\begin{abstract}
In this article, the iteration transform method is used to evaluate the solution of a fractional-order dark optical soliton, bright optical soliton, and periodic solution of the nonlinear Schrödinger equations. The Caputo operator describes the fractional-order derivatives. The solutions of some illustrative examples are presented to show the validity of the proposed technique without using any polynomials. The proposed method provides the series form solutions, which converge to the exact results. Using the present methodology, the solutions of fractional-order problems as well as integral-order problems are calculated. The present method has less computational costs and a higher rate of convergence. Therefore, the suggested algorithm is constructive to solve other fractional-order linear and nonlinear partial differential equations.
\end{abstract}

Keywords: Fiber optics; nonlinear Schrödinger equations; fractional calculus; iterative transform method; analytic solution

\section{Introduction}

The nonlinear Schrödinger equation (NLSE) is a significant equation that arises in a wide range of scientific purposes, including optical fiber communication systems, quantum mechanics, thermodynamics, ocean acoustic performance, biomedical dynamics, and quantum physics [1-3]. Approaches give the NLSE with steep spatial and temporal gradients. This NLSE is constructed from Maxwell's electromagnetic equation using a multiscale perturbation evaluation. NLSEs are commonly used in the literature on shallow-water waves, quantum mechanics, Bose-Einstein condensates, ocean waves, optical solitons, and other fields, with many types of nonlinearity that depend on the background in which they are investigated [4-7]. In the sense that the soliton maintains its amplitude, optical solitons in fibers are pulses that spread-like solitons in shallow water media, 
where interactions of solitons are fully elastic-velocity and shape after nonlinear collisions and the interactions between them influence only the phase shifts. A prototypical integrable scheme is the nonlinear NLS equation, which provides various soliton solutions and infinitely numerous conservation laws. Optical solitons in polarisation-preserving fibers have been commonly used. The complex Ginzburg-Landau equation (CGLE) and many other models were studied with the nonlinear Schrödinger equation [8-10]. In this sense, a deluge of findings has emerged from these as well as other such models.

Optical soliton is an important research area in the field of nonlinear fibre, resulting in a compromise between the self pulse modulation and group velocity dispersion. Optical solitons are acquiring in-depth active research, according to their impressive stability properties and technical features [10]. The decision of optical solitons provides valuable information on the mechanism of complex visual methodologies modeled by the NLSEs.

Several NLSEs were analyzed from the viewpoint of the optical soliton, where different analytical techniques such as the inverse scattering approach illustrated full integrability. However, the two types of nonlinear equations are the most widely utilized ones, i.e.,

$$
i D_{\psi}^{\phi} v+\frac{1}{2} v_{\Im \Im}+v|v|^{2}=0, \quad i=\sqrt{-1}
$$

and

$$
i D_{\psi}^{\phi} v-\frac{1}{2} v_{\Im \Im}+v|v|^{2}=0, \quad i=\sqrt{-1}
$$

used for the normal dispersion regime and the anomalous dispersion regime, respectively. The dynamic function $v(\Im, \psi)$, with $\Im$ as the parameter of the longitudinal variable and $\psi$ acting as the instant of time co-moving. Equation (1) gives a bright soliton approach, which takes a bell-shaped figure and spreads out undistorted for arbitrary long distances, without any shift in shape. However, the NLSEs (Equation (2)) provide dark solitons results, which are behaviors of the nonlinearity of fiber in the normal dispersion regime. Dark solitons are also classified as topological optical solitons in nonlinear optics. The two models (Equations (1) and (2)) emerged widely in the sense of its studies. Several systematic approaches have been utilized for further research on these equations. The explicit approaches of nonlinear integrable equations are significant for their broad applicability in the explanation of natural processes and nonlinear optics [1-11]. In this study, we intend to investigate the above-mentioned NLSEs, each of which contains a detuning term, given as

$$
i D_{\psi}^{\phi} v+v_{\Im \Im}+\alpha v|v|^{2}-\beta v=0, \quad i=\sqrt{-1},
$$

and

$$
i D_{\psi}^{\phi} v-v_{\Im \Im}+\alpha v|v|^{2}-\beta v=0, \quad i=\sqrt{-1},
$$

where there are nonzero constants of $\alpha$ and $\beta$. The time evolution of disturbances in the unstable domain is represented in these two equations. Recently, studies of optical solitons [12-14] have flourished quickly. Some effective techniques have been used to research the properties of pulse development in the area of nonlinear optics.

In 2006, Daftardar-Gejji and Jafari proposed a new iterative method to seek numerical analysis of nonlinear equations $[15,16]$. Jafari et al. first implemented Laplace transformation in the iterative technique and suggested a new direct technique called the iterative Laplace transformation method (ILTM) [17] to search for numerical results of a scheme of FPDEs. ILTM to solve linear and nonlinear partial differential equations such as Fokker-Planck equations [18], Zakharov-Kuznetsov equations [19], the Fornberg-Whitham equation [20], etc. In this article, the iterative method is modified with the new Elzaki transformation; the new method is called the iterative transform method. The iterative transform method is used to evaluate the solution of fractional-order of the dark optical soliton, bright optical soliton, and periodic solution of the proposed nonlinear Schrödinger equations. 
The Caputo operator describes the fractional-order derivatives. The solution of some illustrative examples is presented to show the validity of the proposed technique. Using the present methodology, the solutions of fractional-order problems as well as integral-order problems are calculated. The present method has less computational costs and a higher rate of convergence. Therefore, the suggested algorithm is therefore constructive to solve other fractional-order linear and nonlinear partial differential equations.

\section{Basic Definitions}

Definition 1. The operator $D^{\phi}$ of order $\phi$ in the Abel-Riemann sense is given as [21-23]

$$
D^{\phi} v(\Im)=\left\{\begin{array}{l}
\frac{d^{j}}{d \Im^{j}}, \quad \phi=j \\
\frac{1}{\Gamma(j-\phi)} \frac{d}{d \Im^{j}} \int_{0}^{\Im} \frac{v(\psi)}{(\Im-\psi)^{\psi-j+1}} d t, \quad j-1<\phi<j
\end{array}\right.
$$

where $j \in Z^{+}, \psi \in R^{+}$, and

$$
D^{-\phi} v(\Im)=\frac{1}{\Gamma(\psi)} \int_{0}^{\Im}(\Im-\phi)^{\psi-1} v(\phi) d \phi, \quad 0<\psi \leq 1 .
$$

Definition 2. The Abel-Riemann fractional-order integration operator $J^{\psi}$ is given as [21-23]

$$
J^{\psi} \nu(\Im)=\frac{1}{\Gamma(\psi)} \int_{0}^{\Im}(\Im-\phi)^{\psi-1} v(\phi) d \phi, \phi>0, \quad \psi>0 .
$$

Some properties of the operator:

$$
\begin{aligned}
J^{\psi} \phi^{j} & =\frac{\Gamma(j+1)}{\Gamma(j+\psi+1)} \phi^{j+\psi} \\
D^{\psi} \phi^{j} & =\frac{\Gamma(j+1)}{\Gamma(j-\psi+1)} \phi^{j-\psi}
\end{aligned}
$$

Definition 3. The operator $D^{\psi}$ of fractional-order $\psi$ in the Caputo sense is given as [21-23]

$$
{ }^{C} D^{\psi} v(\Im)=\left\{\begin{array}{l}
\frac{1}{\Gamma(j-\psi)} \int_{0}^{\Im} \frac{v^{j}(\phi)}{(\Im-\phi)^{\psi-j+1}} d \phi, j-1<\psi<j, \\
\frac{d^{j}}{d t^{j}} v(\Im), \quad \psi=j .
\end{array}\right.
$$

\section{Definition 4.}

$$
\begin{aligned}
& J_{\phi}^{\psi} D_{\phi}^{\psi} g(\phi)=g(\phi)-\sum_{k=0}^{m} g^{k}\left(0^{+}\right) \frac{\phi^{k}}{k !}, \text { for } \phi>0, \text { and }-1<\psi \leq j, j \in N . \\
& D_{\phi}^{\psi} J_{\phi}^{\psi} g(\phi)=g(\phi)
\end{aligned}
$$

\section{Fundamental Concept of the Elzaki Transform}

A new transformation called the Elzaki transformation described for the exponential order function that we find in the $A$ set is described by [21-23]:

$$
A=\left\{g(\psi): \ni\left|M, k_{1}, K_{2}>0,\right| g(\psi) \mid<M e^{\frac{|\psi|}{k_{j}}}, i f(\psi) \in(-1)^{j} \times[0, \infty) .\right.
$$


The constant $M$ must be a finite number; $k 1$ and $k 2$ are finite or infinite for a given function in the set. The Elzaki transformation is described through the integral equation

$$
\mathrm{E}[g(\psi)]=T(s)=s \int_{0}^{\infty} g(\psi) e^{\frac{-\psi}{s}} d \psi, \quad \psi \geq 0, k_{1} \leq s \leq k_{2} .
$$

We can achieve the following result from the description and the basic analyses

$$
\begin{aligned}
& \mathrm{E}\left[\psi^{n}\right]=n ! s^{n+2}, \\
& \mathrm{E}\left[g^{\prime}(\psi)\right]=\frac{T(s)}{s}-s g(0), \\
& \mathrm{E}\left[g^{\prime \prime}(\psi)\right]=\frac{T(s)}{s^{2}}-g(0)-s g^{\prime}(0), \\
& \mathrm{E}\left[g^{(n)}(\psi)\right]=\frac{T(s)}{s^{n}}-\sum_{k=0}^{n-1} s^{2-n+k} g^{(k)}(0) .
\end{aligned}
$$

Theorem 1. If $T(s)$ is the Elzaki transformation of $(\psi)$, the Elzaki transformation of the Riemann-Liouville derivative can be taken into consideration as follows [21-23]:

$$
E\left[D^{\phi} g(\psi)\right]=s^{-\phi}\left[T(s)-\sum_{k=1}^{n}\left\{D^{\phi-k} g(0)\right\}\right] ;-1<n-1 \leq \phi<n
$$

Proof. Let us take the Laplace transform of

$$
\begin{gathered}
g^{\prime}(\psi)=\frac{d}{d \psi} g(\psi) \\
L\left[D^{\phi} g(\psi)\right]=S^{\phi} T(s)-\sum_{k=0}^{n-1} s^{k}\left[D^{\phi-k-1} g(0)\right] \\
=s^{\phi} T(s)-\sum_{k=0}^{n-1} s^{k-1}\left[D^{\phi-k} g(0)\right]=s^{\phi} T(s)-\sum_{k=0}^{n-1} s^{k-2}\left[D^{\phi-k} g(0)\right] \\
=s^{\phi} T(s)-\sum_{k=0}^{n-1} \frac{1}{s^{-k+2}}\left[D^{\phi-k} g(0)\right]=s^{\phi} T(s)-\sum_{k=0}^{n-1} \frac{1}{s^{\phi-k+2-\phi}}\left[D^{\phi-k} g(0)\right] \\
=s^{\phi} T(s)-\sum_{k=0}^{n-1} s^{\phi} \frac{1}{s^{\phi-k+2}}\left[D^{\phi-k} g(0)\right] \\
L\left[D^{\phi} g(\psi)\right]=s^{\phi}\left[T(s)-\sum_{k=0}^{n-1}\left(\frac{1}{s}\right)^{\phi-k+2}\left[D^{\phi-k} g(0)\right]\right]
\end{gathered}
$$

Therefore, when we put $\frac{1}{s}$ for $s$, the Elzaki transform of the fractional-order of $g(\psi)$ as below:

$$
E\left[D^{\phi} g(\psi)\right]=s^{-\phi}\left[T(s)-\sum_{k=0}^{n}(s)^{\phi-k+2}\left[D^{\phi-k} g(0)\right]\right]
$$

Definition 5. The Caputo fractional-order of the Elzaki transformation using Theorem 1 is given as:

$$
E\left[D_{\psi}^{\phi} g(\psi)\right]=s^{-\phi} E[g(\psi)]-\sum_{k=0}^{j-1} s^{2-\phi+k} g^{(k)}(0), \text { where } j-1<\phi<j .
$$




\section{Iterative Transform Method (ITM) Idea}

In this part of the paper, we will give the general idea of the recently developed ITM which is used to investigate the system of nonlinear fractional PDEs. Let us take the general fractional PDEs of the form

$$
D_{t}^{\phi} v(\Im, \psi)+N v(\Im, \psi)+M v(\Im, \psi)=h(\Im, \psi), \quad n \in N, n-1<\phi \leq n,
$$

where $N$ and $M$ are linear and nonlinear operators. Furthermore, the initial condition is given by the following

$$
v^{k}(\Im, 0)=g_{k}(\Im), \quad k=0,1,2, \ldots, n-1,
$$

where $\Im$ such that $n-1<\Im \leq n$ is the order of the Caputo fractional operator $D_{t}^{\phi} v(\Im, \psi)$. In the above PDEs, $h(\Im, \psi)$ is the source function. Next, we will apply the Elzaki transform of Equation (7) and obtain the following

$$
E\left[D_{\psi}^{\phi} v(\Im, \psi)\right]+E[N v(\Im, \psi)+M v(\Im, \psi)]=E[h(\Im, \psi)] .
$$

Further simplification through Elzaki differentiation leads to

$$
E[v(\Im, \psi)]=\sum_{k=0}^{m-1} s^{2-\phi+k} u^{(k)}(\Im, 0)+s^{\phi} E[h(\Im, \psi)]-s^{\phi} E[N v(\Im, \psi)+M v(\Im, \psi)] ;
$$

the inverse Elzaki transform converts Equation (10) into

$$
v(\Im, \psi)=E^{-1}\left[\left(\sum_{k=0}^{m-1} s^{2-\phi+k} u^{k}(\Im, 0)+s^{\phi} E[h(\Im, \psi)]\right)\right]-E^{-1}\left[s^{\phi} E[M v(\Im, \psi)+M v(\Im, \psi)]\right] .
$$

As through the iterative technique, we have

$$
v(\Im, \psi)=\sum_{i=0}^{\infty} v_{i}(\Im, \psi) .
$$

Furthermore, the operator $\mathrm{N}$ is linear; therefore, if

$$
N\left(\sum_{i=0}^{\infty} v_{i}(\Im, \psi)\right)=\sum_{i=0}^{\infty} N\left[v_{i}(\Im, \psi)\right]
$$

and the operator $\mathrm{M}$ is nonlinear, we have the following:

$$
M\left(\sum_{i=0}^{\infty} v_{i}(\Im, \psi)\right)=M\left[v_{0}(\Im, \psi)\right]+\sum_{i=1}^{\infty}\left\{M\left(\sum_{k=0}^{i} v_{k}(\Im, \psi)\right)-M\left(\sum_{k=0}^{i-1} v_{k}(\Im, \psi)\right)\right\} .
$$

Putting Equations (12)-(14) in Equation (11), we get the following:

$$
\begin{aligned}
& \sum_{i=0}^{\infty} v_{i}(\Im, \psi)=E^{-1}\left[s^{\phi}\left(\sum_{k=0}^{m-1} s^{2-\Im+k} u^{k}(\Im, 0)+E[h(\Im, \psi)]\right)\right]-E^{-1}\left[s^{\phi} E\right. \\
& \left.\left[\sum_{i=0}^{\infty} N\left[v_{i}(\Im, \psi)\right]+M\left[v_{0}(\Im, \psi)\right]+\sum_{i=1}^{\infty}\left\{M\left(\sum_{k=0}^{i} v_{k}(\Im, \psi)-N\left(\sum_{k=0}^{i-1} v_{k}(\Im, \psi)\right)\right)\right\}\right]\right] .
\end{aligned}
$$

We define the iterative formula mentioned below with applied equations:

$$
v_{0}(\Im, \psi)=E^{-1}\left[s^{\phi}\left(\sum_{k=0}^{m-1} s^{2-\Im+k} u^{k}(\Im, 0)+s^{\phi} E(g(\Im, \psi))\right)\right],
$$




$$
\begin{gathered}
v_{1}(\Im, \psi)=-E^{-1}\left[s^{\phi} E\left[N\left[v_{0}(\Im, \psi)\right]+M\left[v_{0}(\Im, \psi)\right]\right]\right. \\
v_{m+1}(\Im, \psi)=-E^{-1}\left[s^{\phi} L\left[N\left(v_{m}(\Im, \psi)\right)-\left\{M\left(\sum_{k=0}^{m} v_{k}(\Im, \psi)\right)-M\left(\sum_{k=0}^{m-1} v_{k}(\Im, \psi)\right)\right\}\right]\right],
\end{gathered}
$$

$m \geq 1$

Finally, Equations (7) and (8) provide the $m$-term solution in series form is given by

$$
v(\Im, \psi) \cong v_{0}(\Im, \psi)+v_{1}(\Im, \psi)+v_{2}(\Im, \psi)+\ldots . .,+v_{m}(\Im, \psi), \quad m=1,2, \ldots
$$

\section{Applications of the Proposed Method}

In this part of the paper, we determine the solution of NLSEs in the normal dispersion regime through the proposed ITM to inspect its solution with a detuning term. It has been proved that the proposed method is a suitable analytical technique that provides more accurate results for nonlinear FPDEs. Let us take the following fractional Schrödinger equation

$$
\frac{\partial^{\phi} v(\Im, \psi)}{\partial^{\phi} \psi}=-i\left[\frac{\partial^{2} v(\Im, \psi)}{\partial \Im^{2}}-\alpha v(\Im, \psi)|v(\Im, \psi)|^{2}+\beta v(\Im, \psi)\right]
$$

where the fractional-order $\phi$ is such that $0<\phi \leq 1$ while $\alpha$ and $\beta$ are nonzero constants.

\subsection{Dark Optical Soliton Analysis}

To calculate the dark optical soliton results, we take the above Equation (20) with the following initial condition

$$
v_{0}(\Im, 0)=\mu \tanh \left(\mu \sqrt{\frac{\alpha}{2} \Im}\right)
$$

Applying Elzaki transformation to Equation (20), we have

$$
\begin{gathered}
\frac{1}{\mathrm{~s}^{\phi}} E[v(\Im, \psi)]-\sum_{k=0}^{m-1} v_{(k)}(\Im, 0) \mathrm{s}^{2-\phi+k}=E\left[-i\left(\frac{\partial^{2} v(\Im, \psi)}{\partial \Im^{2}}-\alpha v^{2}(\Im, \psi) \bar{v}(\Im, \psi)+\beta v(\Im, \psi)\right)\right], \\
\frac{1}{\mathrm{~s}^{\phi}} E[v(\Im, \psi)]=v_{(0)}(\Im, 0) \mathrm{s}^{2-\phi}+E\left[-i\left(\frac{\partial^{2} v(\Im, \psi)}{\partial \Im^{2}}-\alpha v^{2}(\Im, \psi) \bar{v}(\Im, \psi)+\beta v(\Im, \psi)\right)\right], \\
E[v(\Im, \psi)]=\mathrm{s}^{2} u(\Im, 0)+\mathrm{s}^{\phi}\left[-i\left(\frac{\partial^{2} v(\Im, \psi)}{\partial \Im^{2}}-\alpha v^{2}(\Im, \psi) \bar{v}(\Im, \psi)+\beta v(\Im, \psi)\right)\right] .
\end{gathered}
$$

Using inverse Elzaki transformation of Equation (22), we get

$$
v(\Im, \psi)=E^{-1}\left[s^{2} u(\Im, 0)\right]+E^{-1}\left[-i s^{\phi} E\left(\frac{\partial^{2} v(\Im, \psi)}{\partial \Im^{2}}-\alpha v^{2}(\Im, \psi) \bar{v}(\Im, \psi)+\beta v(\Im, \psi)\right)\right] .
$$

Applying the proposed analytical technique, we get

$$
\begin{aligned}
& v_{0}(\Im, 0)=\mu \tanh \left(\mu \sqrt{\frac{\alpha}{2}}\right) \\
& v_{1}(\Im, \psi)=E^{-1}\left[s^{\phi}\left\{E(-i)\left(\frac{\partial^{2} v_{0}(\Im, \psi)}{\partial \Im^{2}}-\alpha v_{0}^{2}(\Im, \psi) \bar{v}_{0}(\Im, \psi)+\beta v_{0}(\Im, \psi)\right)\right\}\right], \\
& v_{1}(\Im, \psi)=\mu \tanh \left(\mu \sqrt{\frac{\alpha}{2}} \Im\right) i\left(\alpha \mu^{2}-\beta\right) \frac{\psi^{\phi}}{\Gamma(\phi+1)} .
\end{aligned}
$$




$$
\begin{gathered}
v_{2}(\Im, \psi)=E^{-1}\left[s^{\phi}\left\{E(-i)\left(\frac{\partial^{2} v_{1}(\Im, \psi)}{\partial \Im^{2}}-\alpha v_{1}^{2}(\Im, \psi) \bar{v}_{1}(\Im, \psi)+\beta v_{1}(\Im, \psi)\right)\right\}\right], \\
v_{2}(\Im, \psi)=\mu \tanh \left(\mu \sqrt{\left.\frac{\alpha}{2} \Im\right) i\left(\alpha \mu^{2}-\beta\right)^{2} \frac{\psi^{2 \phi}}{\Gamma(2 \phi+1)} .}\right. \\
v_{3}(\Im, \psi)=E^{-1}\left[s^{\phi}\left\{E(-i)\left(\frac{\partial^{2} v_{2}(\Im, \psi)}{\partial \Im^{2}}-\alpha v_{2}^{2}(\Im, \psi) \bar{v}_{2}(\Im, \psi)+\beta v_{2}(\Im, \psi)\right)\right\}\right], \\
v_{3}(\Im, \psi)=-\mu \tanh \left(\mu \sqrt{\frac{\alpha}{2}}\right) i\left(\alpha \mu^{2}-\beta\right)^{3} \frac{\psi^{3 \phi}}{\Gamma(3 \phi+1)} . \\
\vdots \\
v_{n}(\Im, \psi)=E^{-1}\left[s^{\phi}\left\{E(-i)\left(\frac{\partial^{2} v_{n-1}(\Im, \psi)}{\partial \Im^{2}}-\alpha v_{n-1}^{2}(\Im, \psi) \bar{v}_{n-1}(\Im, \psi)+\beta v_{n-1}(\Im, \psi)\right)\right\}\right], \\
v_{n}(\Im, \psi)=\mu \tanh \left(\mu \sqrt{\frac{\alpha}{2}} \Im\right)\left(\left(i\left(\alpha \mu^{2}-\beta\right)\right)^{n} \frac{\psi^{n \phi}}{\Gamma(n \phi+1)}\right), n \geq 0 .
\end{gathered}
$$

The series form solution is given as

$$
v(\Im, \psi)=v_{0}(\Im, \psi)+v_{1}(\Im, \psi)+v_{2}(\Im, \psi)+v_{3}(\Im, \psi)+\cdots v_{n}(\Im, \psi)
$$

Therefore the approximate solution of equation for the first $n$ is given as

$$
\begin{aligned}
& v(\Im, \psi)=i \mu \tanh \left(\mu \sqrt{\frac{\alpha}{2}}\right)\left(1+\left(\alpha \mu^{2}-\beta\right) \frac{\psi^{\phi}}{\Gamma(\phi+1)}-\left(\alpha \mu^{2}-\beta\right)^{2} \frac{\psi^{2 \phi}}{\Gamma(2 \phi+1)}-\right. \\
&\left.\left(\alpha \mu^{2}-\beta\right)^{3} \frac{\psi^{3 \phi}}{\Gamma(3 \phi+1)}+\cdots+\left(\left(\alpha \mu^{2}-\beta\right)^{n} \frac{\psi^{n \phi}}{\Gamma(n \phi+1)}\right)\right) .
\end{aligned}
$$

In the case $\phi=1$,

$$
\begin{gathered}
\left.v(\Im, \psi)=\mu \tanh \left(\mu \sqrt{\frac{\alpha}{2}}\right)\right)\left(1+\left(\alpha \mu^{2}-\beta\right) \psi-\frac{1}{2 !}\left(\alpha \mu^{2}-\beta\right)^{2} \psi^{2}+\cdots+\left(\left(\frac{1}{n !}\left(i\left(\alpha \mu^{2}-\beta\right)\right)^{n} \psi^{n}\right)\right) .\right. \\
v(\Im, \psi)=\mu \operatorname{sech}\left(\mu \sqrt{-\frac{\alpha}{2} \Im}\right) e^{i\left(\frac{\alpha}{2} \mu^{2}-\beta\right) \psi} .
\end{gathered}
$$

The exact result is

$$
u(\Im, 0)=\mu \operatorname{csch}\left(\mu \sqrt{\frac{\alpha}{2}} \Im\right)
$$

we get the singular soliton solution given by

$$
v(\Im, \psi)=\mu \operatorname{csch}\left(\mu \sqrt{\frac{\alpha}{2}} \Im\right) e^{-i\left(\frac{\alpha}{2} \mu^{2}-\beta\right) \psi}
$$

\subsection{Bright Optical Soliton Analysis}

To calculate the dark optical soliton analysis, we take Equation (20) with the following initial condition:

$$
v_{0}(\Im, 0)=\mu \tan \left(\mu \sqrt{\frac{\alpha}{2}} \Im\right)
$$


First, applying Elzaki transformation, we get

$$
\begin{gathered}
\frac{1}{\mathrm{~s}^{\phi}} E[v(\Im, \psi)]-\sum_{k=0}^{m-1} v^{(k)}(\Im, 0) \mathrm{s}^{2-\phi+k}=E\left[-i\left(\frac{\partial^{2} v(\Im, \psi)}{\partial \Im^{2}}-\alpha v^{2}(\Im, \psi) \bar{v}(\Im, \psi)+\beta v(\Im, \psi)\right)\right], \\
\frac{1}{\mathrm{~s}^{\phi}} E[v(\Im, \psi)]=v_{0}(\Im, 0) \mathrm{s}^{2-\phi}+E\left[-i\left(\frac{\partial^{2} v(\Im, \psi)}{\partial \Im^{2}}-\alpha v^{2}(\Im, \psi) \bar{v}(\Im, \psi)+\beta v(\Im, \psi)\right)\right], \\
E[v(\Im, \psi)]=\mathrm{s}^{2} u(\Im, 0)+\mathrm{s}^{\phi}\left[-i\left(\frac{\partial^{2} v(\Im, \psi)}{\partial \Im^{2}}-\alpha v^{2}(\Im, \psi) \bar{v}(\Im, \psi)+\beta v(\Im, \psi)\right)\right] .
\end{gathered}
$$

Elzaki transformation converts the above equation into

$$
v(\Im, \psi)=E^{-1}\left[\mathrm{~s}^{2} u(\Im, 0)\right]+E^{-1}\left[-i \mathrm{~s}^{\phi} E\left(\frac{\partial^{2} v(\Im, \psi)}{\partial \Im^{2}}-\alpha v^{2}(\Im, \psi) \bar{v}(\Im, \psi)+\beta v(\Im, \psi)\right)\right] .
$$

By applying the proposed analytic technique, we have the following:

$$
\begin{gathered}
v_{0}(\Im, \psi)=\mu \tan \left(\mu \sqrt{\frac{\alpha}{2} \Im}\right) \\
v_{1}(\Im, \psi)=E^{-1}\left[-i s^{\phi} E\left\{\frac{\partial^{2} v_{0}(\Im, \psi)}{\partial \Im^{2}}-\alpha v_{0}^{2}(\Im, \psi) \bar{v}_{0}(\Im, \psi)+\beta v_{0}(\Im, \psi)\right\}\right], \\
v_{1}(\Im, \psi)=\mu \tan \left(\mu \sqrt{\frac{\alpha}{2} \Im}\right)\left(-i\left(\alpha \mu^{2}+\beta\right) \frac{\psi^{\phi}}{\Gamma(\phi+1)}\right) . \\
v_{2}(\Im, \psi)=E^{-1}\left[-i \mathbf{s}^{\phi} E\left\{\frac{\partial^{2} v_{1}(\Im, \psi)}{\partial \Im^{2}}-\alpha v_{1}^{2}(\Im, \psi) \bar{v}_{1}(\Im, \psi)+\beta v_{1}(\Im, \psi)\right\}\right], \\
v_{2}(\Im, \psi)=\mu \operatorname{sech}\left(\mu \sqrt{\left.-\frac{\alpha}{2} \Im\right)\left(i\left(\frac{\alpha}{2} \mu^{2}-\beta\right)^{2} \frac{\psi^{2 \phi}}{\Gamma(2 \phi+1)}\right),}\right. \\
v_{3}(\Im, \psi)=E^{-1}\left[-i \mathrm{~s}^{\phi} E\left\{\frac{\partial^{2} v_{2}(\Im, \psi)}{\partial \Im^{2}}-\alpha v_{2}^{2}(\Im, \psi) \bar{v}_{2}(\Im, \psi)+\beta v_{2}(\Im, \psi)\right\}\right], \\
v_{3}(\Im, \psi)=\mu \operatorname{sech}\left(\mu \sqrt{\left.-\frac{\alpha}{2} \Im\right)\left(-i\left(\frac{\alpha}{2} \mu^{2}-\beta\right)^{3} \frac{\psi^{3 \phi}}{\Gamma(3 \phi+1)}\right) .}\right. \\
v_{n}(\Im, \psi)=E^{-1}\left[-i \mathrm{~s}^{\phi} E\left\{\frac{\partial^{2} v_{n-1}(\Im, \psi)}{\partial \Im^{2}}-\alpha v_{n-1}^{2}(\Im, \psi) \bar{v}_{n-1}(\Im, \psi)+\beta v_{n-1}(\Im, \psi)\right\}\right], \\
v_{n}(\Im, \psi)=\mu \operatorname{sech}\left(\mu \sqrt{\frac{\alpha}{2}} \Im\right)\left(i\left(\frac{\alpha}{2} \mu^{2}-\beta\right)^{n} \frac{\psi^{n \phi}}{\Gamma(n \phi+1)}\right), n \geq 0 .
\end{gathered}
$$

Finally, the analytical solution of our problem in series form is

$$
v(\Im, \psi)=v_{0}(\Im, \psi)+v_{1}(\Im, \psi)+v_{2}(\Im, \psi)+v_{3}(\Im, \psi)+\cdots v_{n}(\Im, \psi) .
$$

Therefore, the approximate solution of the equation for the first $n$ is given as

$$
\begin{aligned}
& v(\Im, \psi)=i \mu \operatorname{sech}\left(\mu \sqrt{-\frac{\alpha}{2}}\right)\left(1+\left(\frac{\alpha}{2} \mu^{2}-\beta\right) \frac{\psi^{\phi}}{\Gamma(\phi+1)}-\left(\frac{\alpha}{2} \mu^{2}-\beta\right)^{2} \frac{\psi^{2 \phi}}{\Gamma(2 \phi+1)}\right. \\
&\left.+\cdots+\left(\frac{\alpha}{2} \mu^{2}-\beta\right)^{n} \frac{\psi^{n \phi}}{\Gamma(n \phi+1)}\right)
\end{aligned}
$$


If $\phi=1$, then

$$
\begin{gathered}
v(\Im, \psi)=\mu \operatorname{sech}\left(\mu \sqrt{-\frac{\alpha}{2}} \Im\right)\left(1+\left(\frac{\alpha}{2} \mu^{2}-\beta\right) \psi-\frac{1}{2 !}\left(\frac{\alpha}{2} \mu^{2}-\beta\right)^{2} \psi^{2}+\cdots+\frac{1}{n !}\left(i\left(\frac{\alpha}{2} \mu^{2}-\beta\right)\right)^{n} \psi^{n}\right) . \\
v(\Im, \psi)=\mu \operatorname{csch}\left(\mu \sqrt{\frac{\alpha}{2}} \Im\right) e^{-i\left(\frac{\alpha}{2} \mu^{2}-\beta\right) \psi}
\end{gathered}
$$

which is the exact solution. In the same way, if we take the initial condition

$$
u(\Im, 0)=\mu \operatorname{csch}\left(\mu \sqrt{\frac{\alpha}{2} \Im}\right)
$$

we can prove the singular soliton solution following the same steps:

$$
v(\Im, \psi)=\mu \operatorname{csch}\left(\mu \sqrt{\frac{\alpha}{2}} \Im\right) e^{-i\left(\frac{\alpha}{2} \mu^{2}-\beta\right) \psi} .
$$

\subsection{Periodic Results}

For the periodic solution of our problem given in Equation (20), we take the initial condition as

$$
v_{0}(\Im, 0)=\mu \tan \left(\mu \sqrt{\frac{\alpha}{2}} \Im\right)
$$

First, applying Elzaki transformation, we get

$$
\begin{gathered}
\frac{1}{\mathrm{~s}^{\phi}} E[v(\Im, \psi)]-\sum_{k=0}^{m-1} v^{(k)}(\Im, 0) \mathrm{s}^{2-\phi+k}=E\left[-i\left(\frac{\partial^{2} v(\Im, \psi)}{\partial \Im^{2}}-\alpha v^{2}(\Im, \psi) \bar{v}(\Im, \psi)+\beta v(\Im, \psi)\right)\right], \\
\frac{1}{\mathrm{~s}^{\phi}} E[v(\Im, \psi)]=v_{0}(\Im, 0) \mathrm{s}^{2-\phi}+E\left[-i\left(\frac{\partial^{2} v(\Im, \psi)}{\partial \Im^{2}}-\alpha v^{2}(\Im, \psi) \bar{v}(\Im, \psi)+\beta v(\Im, \psi)\right)\right], \\
E[v(\Im, \psi)]=\mathrm{s}^{2} u(\Im, 0)+\mathrm{s}^{\phi}\left[-i\left(\frac{\partial^{2} v(\Im, \psi)}{\partial \Im^{2}}-\alpha v^{2}(\Im, \psi) \bar{v}(\Im, \psi)+\beta v(\Im, \psi)\right)\right] .
\end{gathered}
$$

Then, applying inverse Elzaki transformation, we obtain

$$
v(\Im, \psi)=E^{-1}\left[\mathrm{~s}^{2} u(\Im, 0)\right]+E^{-1}\left[-i \mathrm{~s}^{\phi} E\left(\frac{\partial^{2} v(\Im, \psi)}{\partial \Im^{2}}-\alpha v^{2}(\Im, \psi) \bar{v}(\Im, \psi)+\beta v(\Im, \psi)\right)\right] .
$$

By applying the proposed analytic technique, we have the following:

$$
\begin{aligned}
& v_{0}(\Im, 0)=\mu \tan \left(\mu \sqrt{\frac{\alpha}{2}} \Im\right), \\
& v_{1}(\Im, \psi)=E^{-1}\left[-i s^{\phi} E\left\{\left(\frac{\partial^{2} v_{0}(\Im, \psi)}{\partial \Im^{2}}-\alpha v_{0}^{2}(\Im, \psi) \bar{v}_{0}(\Im, \psi)+\beta v_{0}(\Im, \psi)\right)\right\}\right], \\
& v_{1}(\Im, \psi)=\mu \tan \left(\mu \sqrt{\frac{\alpha}{2}} \Im\right)\left(-i\left(\alpha \mu^{2}+\beta\right) \frac{\psi^{\phi}}{\Gamma(\phi+1)}\right), \\
& v_{2}(\Im, \psi)=E^{-1}\left[-i s^{\phi} E\left\{\left(\frac{\partial^{2} v_{1}(\Im, \psi)}{\partial \Im^{2}}-\alpha v_{1}^{2}(\Im, \psi) \bar{v}_{1}(\Im, \psi)+\beta v_{1}(\Im, \psi)\right)\right\}\right], \\
& v_{2}(\Im, \psi)=\mu \tan \left(\mu \sqrt{\frac{\alpha}{2}}\right)\left(-i\left(\alpha \mu^{2}+\beta\right)^{2} \frac{\psi^{2 \phi}}{\Gamma(2 \phi+1)}\right),
\end{aligned}
$$




$$
\begin{aligned}
& v_{3}(\Im, \psi)=E^{-1}\left[-i s^{\phi} E\left\{\left(\frac{\partial^{2} v_{2}(\Im, \psi)}{\partial \Im^{2}}-\alpha v_{2}^{2}(\Im, \psi) \bar{v}_{2}(\Im, \psi)+\beta v_{2}(\Im, \psi)\right)\right\}\right], \\
& v_{3}(\Im, \psi)=\mu \tan \left(\mu \sqrt{\frac{\alpha}{2}} \Im\right)\left(i\left(\alpha \mu^{2}+\beta\right)^{3} \frac{\psi^{3 \phi}}{\Gamma(3 \phi+1)}\right) . \\
& v_{n}(\Im, \psi)=E^{-1}\left[-i \mathrm{~s}^{\phi} E\left\{\left(\frac{\partial^{2} v_{n-1}(\Im, \psi)}{\partial \Im^{2}}-\alpha v_{n-1}^{2}(\Im, \psi) \bar{v}_{n-1}(\Im, \psi)+\beta v_{n-1}(\Im, \psi)\right)\right\}\right], \\
& v_{n}(\Im, \psi)=\mu \tan \left(\mu \sqrt{\frac{\alpha}{2}}\right)\left(i\left(\alpha \mu^{2}+\beta\right)^{n} \frac{\psi^{n \phi}}{\Gamma(n \phi+1)}\right), n \geq 0 .
\end{aligned}
$$

Thus, the solution in periodic form is given by

$$
v(\Im, \psi)=v_{0}(\Im, \psi)+v_{1}(\Im, \psi)+v_{2}(\Im, \psi)+v_{3}(\Im, \psi)+\cdots v_{n}(\Im, \psi) .
$$

Therefore, the approximate solution of equation for the first $n$ is given as

$$
\begin{aligned}
v(\Im, \psi)=\mu \tan \left(i \mu \sqrt{\frac{\alpha}{2} \Im}\right) & \left(1+\left(\alpha \mu^{2}-\beta\right) \frac{\psi^{\phi}}{\Gamma(\phi+1)}-\left(\alpha \mu^{2}-\beta\right)^{2} \frac{\psi^{2 \phi}}{\Gamma(2 \phi+1)}\right. \\
& \left.+\cdots+\left(\alpha \mu^{2}-\beta\right)^{n} \frac{\psi^{n \phi}}{\Gamma(n \phi+1)}\right) .
\end{aligned}
$$

If $\phi=1$, then

$$
\begin{gathered}
v(\Im, \psi)=\mu \tan \left(\mu \sqrt{\frac{\alpha}{2}}\right)\left(1+\left(\alpha \mu^{2}-\beta\right) \psi-\frac{1}{2 !}\left(\alpha \mu^{2}-\beta\right)^{2} \psi^{2}+\cdots+\frac{1}{n !} i\left(\alpha \mu^{2}-\beta\right)^{n} \psi^{n}\right) . \\
v(\Im, \psi)=\mu \tan \left(\mu \sqrt{\frac{\alpha}{2}} \Im\right) e^{-i\left(\frac{\alpha}{2} \mu^{2}+\beta\right) \psi,}
\end{gathered}
$$

which is the exact solution. If we take the initial condition

$$
u(\Im, 0)=\mu \cot \left(\mu \sqrt{\frac{\alpha}{2}} \Im\right)
$$

then the singular soliton is obtained as

$$
v(\Im, \psi)=\mu \cot \left(\mu \sqrt{\frac{\alpha}{2}} \Im\right) e^{-i\left(\frac{\alpha}{2} \mu^{2}+\beta\right) \psi} .
$$

\section{NLSEs with Detuning Term in the Anomalous Dispersion Regime}

Here, we will apply ITM to determine the dark optical soliton, bright optical soliton, and periodic solution of the proposed equation with a detuning term in the anomalous dispersion regime. In this case, we take NLSE of the form

$$
\frac{\partial^{\phi} v(\Im, \psi)}{\partial^{\phi} x}=i\left[\frac{\partial^{2} v(\Im, \psi)}{\partial \Im^{2}}+\alpha v(\Im, \psi)|v(\Im, \psi)|^{2}-\beta v(\Im, \psi)\right], \quad 0<\phi<1,
$$

where $\alpha$ and $\beta$ are nonzero constants. We will take different initial conditions with this equation to find different solutions. 


\subsection{Dark Optical Soliton Analysis}

To calculate the dark optical soliton analysis, we take the initial condition

$$
v_{0}(\Im, 0)=\mu \tanh \left(\mu \sqrt{-\frac{\alpha}{2}} \Im\right) .
$$

First of all, we apply Elzaki transformation in the following manner

$$
\begin{gathered}
\frac{1}{\mathrm{~s}^{\phi}} E[v(\Im, \psi)]-\sum_{k=0}^{m-1} v^{(k)}(\Im, 0) \mathrm{s}^{2-\phi+k}=E\left[i\left(\frac{\partial^{2} v(\Im, \psi)}{\partial \Im^{2}}+\alpha v^{2}(\Im, \psi) \bar{v}(\Im, \psi)-\beta v(\Im, \psi)\right)\right], \\
\frac{1}{\mathrm{~s}^{\phi}} E[v(\Im, \psi)]=v_{0}(\Im, 0) \mathrm{s}^{2-\phi}+E\left[i\left(\frac{\partial^{2} v(\Im, \psi)}{\partial \Im^{2}}+\alpha v^{2}(\Im, \psi) \bar{v}(\Im, \psi)-\beta v(\Im, \psi)\right)\right], \\
E[v(\Im, \psi)]=\mathrm{s}^{2} u(\Im, 0)+E\left[i \mathrm{~s}^{\phi}\left(\frac{\partial^{2} v(\Im, \psi)}{\partial \Im^{2}}+\alpha v^{2}(\Im, \psi) \bar{v}(\Im, \psi)-\beta v(\Im, \psi)\right)\right] .
\end{gathered}
$$

Applying inverse Elzaki transformation, we have the following

$$
v(\Im, \psi)=E^{-1}\left[\mathrm{~s}^{2} u(\Im, 0)\right]+E^{-1}\left[-i \mathrm{~s}^{\phi} E\left(\frac{\partial^{2} v(\Im, \psi)}{\partial \Im^{2}}-\alpha v^{2}(\Im, \psi) \bar{v}(\Im, \psi)+\beta v(\Im, \psi)\right)\right] .
$$

Furthermore, when we apply the above iterative technique, we get the following:

$$
\begin{aligned}
& v_{0}(\Im, \psi)=\mu \tanh \left(\mu \sqrt{-\frac{\alpha}{2}} \Im\right) \\
& v_{1}(\Im, \psi)=E^{-1}\left[-i \mathbf{s}^{\phi} E\left\{\frac{\partial^{2} v_{0}(\Im, \psi)}{\partial \Im^{2}}-\alpha v_{0}^{2}(\Im, \psi) \bar{v}_{0}(\Im, \psi)+\beta v_{0}(\Im, \psi)\right\}\right], \\
& v_{1}(\Im, \psi)=\mu \tanh \left(\mu \sqrt{-\frac{\alpha}{2}} \Im\right)\left(-i\left(2 \mu^{2}+\beta\right) \frac{\psi^{\phi}}{\Gamma(\phi+1)}\right) . \\
& v_{2}(\Im, \psi)=E^{-1}\left[-i \mathbf{S}^{\phi} E\left\{\frac{\partial^{2} v_{1}(\Im, \psi)}{\partial \Im^{2}}-\alpha v_{1}^{2}(\Im, \psi) \bar{v}_{1}(\Im, \psi)+\beta v_{1}(\Im, \psi)\right\}\right], \\
& v_{2}(\Im, \psi)=\mu \tanh \left(\mu \sqrt{-\frac{\alpha}{2}} \Im\right)\left(-i\left(2 \mu^{2}+\beta\right)^{2} \frac{\psi^{2 \phi}}{\Gamma(2 \phi+1)}\right) \\
& v_{3}(\Im, \psi)=E^{-1}\left[-i \mathrm{~s}^{\phi} E\left\{\frac{\partial^{2} v_{2}(\Im, \psi)}{\partial \Im^{2}}-\alpha v_{2}^{2}(\Im, \psi) \bar{v}_{2}(\Im, \psi)+\beta v_{2}(\Im, \psi)\right\}\right], \\
& v_{3}(\Im, \psi)=-\mu \tanh \left(\mu \sqrt{-\frac{\alpha}{2}} \Im\right)\left(\left(2 \mu^{2}+\beta\right)^{3} \frac{\psi^{3 \phi}}{\Gamma(3 \phi+1)}\right) . \\
& v_{n}(\Im, \psi)=E^{-1}\left[-i \Phi^{\phi} E\left\{\frac{\partial^{2} v_{n-1}(\Im, \psi)}{\partial \Im^{2}}-\alpha v_{n-1}^{2}(\Im, \psi) \bar{v}_{n-1}(\Im, \psi)+\beta v_{n-1}(\Im, \psi)\right\}\right], \\
& v_{n}(\Im, \psi)=\mu \tanh \left(\mu \sqrt{-\frac{\alpha}{2}} \Im\right)\left(-i\left(2 \mu^{2}+\beta\right)^{n} \frac{\psi^{n \phi}}{\Gamma(n \phi+1)}\right), n \geq 0 .
\end{aligned}
$$

Thus, the analytic solution of the given problem is

$$
v(\Im, \psi)=v_{0}(\Im, \psi)+v_{1}(\Im, \psi)+v_{2}(\Im, \psi)+v_{3}(\Im, \psi)+\cdots v_{n}(\Im, \psi)
$$


Therefore, the approximate solution of equation for the first $n$ is given as

$$
\begin{aligned}
v(\Im, \psi)=\mu \tanh \left(\mu \sqrt{-\frac{\alpha}{2}} \Im\right) & \left(1-i\left(2 \mu^{2}+\beta\right) \frac{\psi^{\phi}}{\Gamma(\phi+1)}-\left(2 \mu^{2}+\beta\right)^{2} \frac{\psi^{2 \phi}}{\Gamma(2 \phi+1)}\right. \\
& \left.+\cdots+i\left(2 \mu^{2}+\beta\right)^{n} \frac{\psi^{n \phi}}{\Gamma(n \phi+1)}\right) .
\end{aligned}
$$

If $\phi=1$ then

$$
\begin{gathered}
v(\Im, \psi)=\mu \tanh \left(\mu \sqrt{-\frac{\alpha}{2}} \Im\right)\left(1-i\left(2 \mu^{2}+\beta\right) \psi-\frac{1}{2 !}\left(2 \mu^{2}+\beta\right)^{2} \psi^{2}+\cdots+\frac{1}{n !} i\left(2 \mu^{2}+\beta\right)^{n} \psi^{n}\right) . \\
v(\Im, \psi)=\mu \operatorname{sech}\left(\mu \sqrt{-\frac{\alpha}{2}} \Im\right) e^{i\left(\frac{\alpha}{2} \mu^{2}-\beta\right) \psi}
\end{gathered}
$$

The exact result is

$$
u(\Im, 0)=\mu \operatorname{csch}\left(\mu \sqrt{\frac{\alpha}{2}} \Im\right)
$$

and we determine the following singular soliton solution by following the same steps:

$$
v(\Im, \psi)=\mu \operatorname{csch}\left(\mu \sqrt{\frac{\alpha}{2}} \Im\right) e^{-i\left(\frac{\alpha}{2} \mu^{2}-\beta\right) \psi} .
$$

\subsection{Bright Optical Soliton Analysis}

To calculate the bright optical soliton analysis, we take the above Equation (58) with the following initial condition:

$$
v_{0}(\Im, 0)=\mu \sqrt{\frac{2}{\alpha}} \operatorname{sech}(\mu, x) .
$$

First, applying Elzaki transformation, we get

$$
\begin{gathered}
\frac{1}{\mathrm{~s}^{\phi}} E[v(\Im, \psi)]-\sum_{k=0}^{m-1} v^{(k)}(\Im, 0) \mathrm{s}^{2-\phi+k}=E\left[i\left(\frac{\partial^{2} v(\Im, \psi)}{\partial \Im^{2}}+\alpha v^{2}(\Im, \psi) \bar{v}(\Im, \psi)-\beta v(\Im, \psi)\right)\right], \\
\frac{1}{\mathrm{~s}^{\phi}} E[v(\Im, \psi)]=v_{0}(\Im, 0) \mathrm{s}^{2-\phi}+E\left[i\left(\frac{\partial^{2} v(\Im, \psi)}{\partial \Im^{2}}+\alpha v^{2}(\Im, \psi) \bar{v}(\Im, \psi)-\beta v(\Im, \psi)\right)\right], \\
E[v(\Im, \psi)]=\mathrm{s}^{2} u(\Im, 0)+E\left[i \mathrm{~s}^{\phi}\left(\frac{\partial^{2} v(\Im, \psi)}{\partial \Im^{2}}+\alpha v^{2}(\Im, \psi) \bar{v}(\Im, \psi)-\beta v(\Im, \psi)\right)\right] .
\end{gathered}
$$

Elzaki transformation converts the above equation into

$$
v(\Im, \psi)=E^{-1}\left[\mathrm{~s}^{2} u(\Im, 0)\right]+E^{-1}\left[i \mathrm{~s}^{\phi} E\left(\frac{\partial^{2} v(\Im, \psi)}{\partial \Im^{2}}+\alpha v^{2}(\Im, \psi) \bar{v}(\Im, \psi)-\beta v(\Im, \psi)\right)\right] .
$$

By applying the proposed analytic technique, we have the following:

$$
\begin{aligned}
& v_{0}(\Im, \psi)=\mu \sqrt{\frac{2}{\alpha}} \operatorname{sech}(\mu \Im) \\
& v_{1}(\Im, \psi)=E^{-1}\left[-i s^{\phi} E\left\{\frac{\partial^{2} v_{0}(\Im, \psi)}{\partial \Im^{2}}-\alpha v_{0}^{2}(\Im, \psi) \bar{v}_{0}(\Im, \psi)+\beta v_{0}(\Im, \psi)\right\}\right], \\
& v_{1}(\Im, \psi)=\mu \sqrt{\frac{2}{\alpha}} \operatorname{sech}(\mu \Im)\left(i\left(\mu^{2}-\beta\right) \frac{\psi^{\phi}}{\Gamma(\phi+1)}\right) .
\end{aligned}
$$




$$
\begin{gathered}
v_{2}(\Im, \psi)=E^{-1}\left[-i \mathrm{~s}^{\phi} E\left\{\frac{\partial^{2} v_{1}(\Im, \psi)}{\partial \Im^{2}}-\alpha v_{1}^{2}(\Im, \psi) \bar{v}_{1}(\Im, \psi)+\beta v_{1}(\Im, \psi)\right\}\right], \\
v_{2}(\Im, \psi)=\mu \sqrt{\frac{2}{\alpha} \operatorname{sech}(\mu \Im)\left(-i\left(\mu^{2}-\beta\right)^{2} \frac{\psi^{2 \phi}}{\Gamma(2 \phi+1)}\right)} \\
v_{3}(\Im, \psi)=E^{-1}\left[-i \mathrm{~s}^{\phi} E\left\{\frac{\partial^{2} v_{2}(\Im, \psi)}{\partial \Im^{2}}-\alpha v_{2}^{2}(\Im, \psi) \bar{v}_{2}(\Im, \psi)+\beta v_{2}(\Im, \psi)\right\}\right], \\
v_{3}(\Im, \psi)=-\mu \sqrt{\frac{2}{\alpha}} \operatorname{sech}(\mu \Im)\left(\left(\mu^{2}-\beta\right)^{3} \frac{\psi^{3 \phi}}{\Gamma(3 \phi+1)}\right) . \\
\vdots \\
v_{n}(\Im, \psi)=E^{-1}\left[-i \mathrm{~s}^{\phi} E\left\{\frac{\partial^{2} v_{n-1}(\Im, \psi)}{\partial \Im^{2}}-\alpha v_{n-1}^{2}(\Im, \psi) \bar{v}_{n-1}(\Im, \psi)+\beta v_{n-1}(\Im, \psi)\right\}\right], \\
v_{n}(\Im, \psi)=\mu \sqrt{\frac{2}{\alpha}} \operatorname{sech}(\mu \Im)\left(i\left(\mu^{2}-\beta\right)^{n} \frac{\psi^{n \phi}}{\Gamma(n \phi+1)}\right), n \geq 0 .
\end{gathered}
$$

The series form solution is given as

$$
v(\Im, \psi)=v_{0}(\Im, \psi)+v_{1}(\Im, \psi)+v_{2}(\Im, \psi)+v_{3}(\Im, \psi)+\cdots v_{n}(\Im, \psi)
$$

Therefore, the approximate solution of equation for the first $n$ is given as

$$
\begin{aligned}
v(\Im, \psi)=\mu \sqrt{\frac{2}{\alpha}} \operatorname{sech}(\mu \Im) & \left(1+i\left(\mu^{2}-\beta\right) \frac{\psi^{\phi}}{\Gamma(\phi+1)}-\left(\mu^{2}-\beta\right)^{2} \frac{\psi^{2 \phi}}{\Gamma(2 \phi+1)}\right. \\
& \left.+\cdots+\left(i\left(\mu^{2}-\beta\right)\right)^{n} \frac{\psi^{n \phi}}{\Gamma(n \phi+1)}\right) .
\end{aligned}
$$

In the case $\phi=1$,

$$
\begin{gathered}
v(\Im, \psi)=\mu \tanh \left(\mu \sqrt{\frac{\alpha}{2}}\right)\left(1+\left(\alpha \mu^{2}-\beta\right) \psi-\frac{1}{2 !}\left(\alpha \mu^{2}-\beta\right)^{2} \psi^{2}+\cdots+\frac{1}{n !}\left(i\left(\alpha \mu^{2}-\beta\right)\right)^{n} \psi^{n}\right) . \\
v(\Im, \psi)=\mu \operatorname{sech}\left(\mu \sqrt{-\frac{\alpha}{2}} \Im\right) e^{i\left(\frac{\alpha}{2} \mu^{2}-\beta\right) \psi} .
\end{gathered}
$$

The exact solution is

$$
u(\Im, 0)=\mu \operatorname{csch}\left(\mu \sqrt{\frac{\alpha}{2}} \Im\right)
$$

and we obtain the following singular soliton solution for the above problem:

$$
v(\Im, \psi)=\mu \operatorname{csch}\left(\mu \sqrt{\frac{\alpha}{2}} \Im\right) e^{-i\left(\frac{\alpha}{2} \mu^{2}-\beta\right) \psi} .
$$

\subsection{Solutions in Periodic Environment}

To determine the periodic solutions, we take the above Equation (58) with the following initial condition:

$$
v_{0}(\Im, \psi)=\mu \sqrt{-\frac{2}{\alpha}} \tan (\mu \Im)
$$


Applying Elzaki transformation to Equation (58), we have

$$
\begin{gathered}
s^{\phi} E\left[\vartheta\left(\ell, \psi_{1}\right)\right]-\sum_{k=0}^{m-1} \vartheta^{(k)}(\ell, 0) s^{2-\phi+k}=E\left[i\left(\frac{\partial^{2} v(\Im, \psi)}{\partial \Im^{2}}+\alpha v^{2}(\Im, \psi) \bar{v}(\Im, \psi)-\beta v(\Im, \psi)\right)\right], \\
s^{\phi} E\left[\vartheta\left(\ell, \psi_{1}\right)\right]=\vartheta^{(0)}(\ell, 0) s^{2}+E\left[-i\left(\frac{\partial^{2} v(\Im, \psi)}{\partial \Im^{2}}-\alpha v^{2}(\Im, \psi) \bar{v}(\Im, \psi)+\beta v(\Im, \psi)\right)\right], \\
E\left[\vartheta\left(\ell, \psi_{1}\right)\right]=\frac{\vartheta(\ell, 0)}{s}+E\left[i s^{\phi}\left(\frac{\partial^{2} v(\Im, \psi)}{\partial \Im^{2}}+\alpha v^{2}(\Im, \psi) \bar{v}(\Im, \psi)-\beta v(\Im, \psi)\right)\right] .
\end{gathered}
$$

Applying inverse Elzaki transformation, we have

$$
\vartheta\left(\ell, \psi_{1}\right)=E^{-1}\left[\frac{\vartheta(\ell, 0)}{s}\right]+E^{-1}\left[i s^{\phi} E\left(\frac{\partial^{2} v(\Im, \psi)}{\partial \Im^{2}}+\alpha v^{2}(\Im, \psi) \bar{v}(\Im, \psi)-\beta v(\Im, \psi)\right)\right] .
$$

By applying the proposed analytic technique, we have the following:

$$
\begin{aligned}
& v_{0}(\Im, \psi)=\mu \tanh \left(\mu \sqrt{\frac{\alpha}{2}}\right) \\
& v_{1}(\Im, \psi)=E^{-1}\left[i s^{\phi} E\left\{\frac{\partial^{2} v_{0}(\Im, \psi)}{\partial \Im^{2}}+\alpha v_{0}^{2}(\Im, \psi) \bar{v}_{0}(\Im, \psi)-\beta v_{0}(\Im, \psi)\right\}\right], \\
& v_{1}(\Im, \psi)=\mu \tanh \left(\mu \sqrt{\frac{\alpha}{2}} \Im\right)\left(i\left(\alpha \mu^{2}-\beta\right) \frac{\psi^{\phi}}{\Gamma(\phi+1)}\right) . \\
& v_{2}(\Im, \psi)=E^{-1}\left[i s^{\phi} E\left\{\frac{\partial^{2} v_{1}(\Im, \psi)}{\partial \Im^{2}}+\alpha v_{1}^{2}(\Im, \psi) \bar{v}_{1}(\Im, \psi)+\beta v_{1}(\Im, \psi)\right\}\right], \\
& v_{2}(\Im, \psi)=\mu \tanh \left(\mu \sqrt{\frac{\alpha}{2}}\right)\left(-i\left(\alpha \mu^{2}-\beta\right)^{2} \frac{\psi^{2 \phi}}{\Gamma(2 \phi+1)}\right) \\
& v_{3}(\Im, \psi)=E^{-1}\left[i s^{\phi} E\left\{\frac{\partial^{2} v_{2}(\Im, \psi)}{\partial \Im^{2}}+\alpha v_{2}^{2}(\Im, \psi) \bar{v}_{2}(\Im, \psi)-\beta v_{2}(\Im, \psi)\right\}\right], \\
& v_{3}(\Im, \psi)=\mu \tanh \left(\mu \sqrt{\frac{\alpha}{2}} \Im\right)\left(-i\left(\alpha \mu^{2}-\beta\right)^{3} \frac{\psi^{3 \phi}}{\Gamma(3 \phi+1)}\right) . \\
& v_{n}(\Im, \psi)=E^{-1}\left[i s^{\phi} E\left\{\frac{\partial^{2} v_{n-1}(\Im, \psi)}{\partial \Im^{2}}+\alpha v_{n-1}^{2}(\Im, \psi) \bar{v}_{n-1}(\Im, \psi)-\beta v(\Im, \psi)\right\}\right], \\
& v_{n}(\Im, \psi)=\mu \tanh \left(\mu \sqrt{\frac{\alpha}{2}} \Im\right)\left(\left(i\left(\alpha \mu^{2}-\beta\right)\right)^{n} \frac{\psi^{n \phi}}{\Gamma(n \phi+1)}\right), n \geq 0 .
\end{aligned}
$$

Therefore, we have the following analytic solution:

$$
v(\Im, \psi)=v_{0}(\Im, \psi)+v_{1}(\Im, \psi)+v_{2}(\Im, \psi)+v_{3}(\Im, \psi)+\cdots v_{n}(\Im, \psi),
$$

Hence, the approximate solution of the equation for the first $n$ is given as

$$
\begin{aligned}
v(\Im, \psi)= & \mu \tanh \left(\mu \sqrt{\frac{\alpha}{2}}\right)\left(1+i\left(\alpha \mu^{2}-\beta\right) \frac{\psi^{\phi}}{\Gamma(\phi+1)}-\left(\alpha \mu^{2}-\beta\right)^{2} \frac{\psi^{2 \phi}}{\Gamma(2 \phi+1)}\right. \\
& \left.+\cdots+\left(i\left(\alpha \mu^{2}-\beta\right)\right)^{n} \frac{\psi^{n \phi}}{\Gamma(n \phi+1)}\right) .
\end{aligned}
$$


In the case $\phi=1$,

$$
\begin{gathered}
v(\Im, \psi)=\mu \tanh \left(\mu \sqrt{\frac{\alpha}{2}}\right)\left(1+i\left(\alpha \mu^{2}-\beta\right) \psi-\frac{1}{2 !}\left(\alpha \mu^{2}-\beta\right)^{2} \psi^{2}+\cdots+\frac{1}{n !}\left(i\left(\alpha \mu^{2}-\beta\right)\right)^{n} \psi^{n}\right) . \\
v(\Im, \psi)=\mu \operatorname{sech}\left(\mu \sqrt{-\frac{\alpha}{2}} \Im\right) e^{i\left(\frac{\alpha}{2} \mu^{2}-\beta\right) \psi} .
\end{gathered}
$$

The exact result is

$$
u(\Im, 0)=\mu \operatorname{csch}\left(\mu \sqrt{\frac{\alpha}{2} \Im}\right) .
$$

The singular soliton result is calculated with the help of the present technique

$$
v(\Im, \psi)=\mu \operatorname{csch}\left(\mu \sqrt{\frac{\alpha}{2}} \Im\right) e^{-i\left(\frac{\alpha}{2} \mu^{2}-\beta\right) \psi} .
$$

\section{Conclusions}

In this paper, we evaluated the fractional-order optical bright and dark solitons for the nonlinear Schrödinger equations with a detuning term in the normal and anomalous dispersive regimes using an iteration transform method. The solutions for specific problems are calculated using the proposed technique. The iteration transform method results are in close contact with the exact solution of the suggested models. The present technique also calculates the solutions of the problems with the fractional-order derivatives. Moreover, the current technique is straightforward and simple, and it carries less computational cost; the suggested method can be modeled to solve other fractional-order partial differential equations.

Author Contributions: Conceptualization, N.A.S. and P.A.; methodology, N.A.S.; software, E.R.E.-Z. and Y.S.H.; validation, J.D.C.; formal analysis, N.A.S. and P.A.; data curation, Y.S.H.; writing-original draft preparation E.R.E.-Z.; writing-review and editing, N.A.S.; supervision, J.D.C.; project administration, P.A.; funding acquisition, J.D.C. All authors have read and agreed to the published version of the manuscript.

Funding: Not applicable.

Acknowledgments: This research was supported by the Basic Science Research Program through the National Research Foundation of Korea (NRF) funded by the Ministry of Education (No. 2017R1D1A1B05030422). This Research was supported by Taif University Researchers Supporting Project Number (TURSP-2020/155), Taif University, Taif, Saudi Arabia.

Conflicts of Interest: The authors have no conflict of interest.

\section{References}

1. Triki, H.; Biswas, A. Dark solitons for a generalized nonlinear Schrödinger equation with parabolic law and dual-power law nonlinearities. Math. Methods Appl. Sci. 2011, 34, 958-962. [CrossRef]

2. Wazwaz, A.M. The integrable time-dependent sine-Gordon with multiple optical kink solutions. Optik 2019, 182, 605-610. [CrossRef]

3. Biswas, A.; Milovic, D. Bright and dark solitons of the generalized nonlinear Schrödinger's equation. Commun. Nonlinear Sci. Numer. Simul. 2010, 15, 1473-1484. [CrossRef]

4. Tala-Tebuea, E.; Djoufack, Z.I.; Fendzi-Donfack, E.; Kenfack-Jiotsa, A.; Kofané, T.C. Exact solutions of the unstable nonlinear Schrödinger equation with the new Jacobi elliptic function rational expansion method and the exponential rational function method. Optik 2016, 127, 11124-11130. [CrossRef]

5. Biswas, A. Conservation laws for optical solitons with anti-cubic and generalized anti-cubic nonlinearities. Optik 2019, 176, 198-201. [CrossRef]

6. Mackenzie, J.A.; Mekwi, W.R. An hr-adaptive method for the cubic nonlinear Schrödinger equation. J. Comput. Appl. Math. 2020, 364, 112320. [CrossRef]

7. Malomed, B. Nonlinear Schrödinger Equations. In Encyclopedia of Nonlinear Science; Scott, A., Ed.; Routledge: New York, NY, UA, 2005; pp. 639-643 
8. Vinoj, M.N.; Kuriakose, V.C. Nonlinear compression of optical solitons. Pramana 2001, 57, 987-1001. [CrossRef]

9. Leblond, H.; Mihalache, D. Models of few optical cycle solitons beyond the slowly varying envelope approximation. Phys. Rep. 2013, 523, 61-126. [CrossRef]

10. Biswas, A.; Arshed, S. Application of semi-inverse variational principle to cubic-quartic optical solitons with kerr and power law nonlinearity. Optik 2018, 172, 847-850. [CrossRef]

11. Wazwaz, A.M. A variety of optical solitons for nonlinear Schrodinger equation with detuning term by the variational iteration method. Optik 2019, 196, 163169. [CrossRef]

12. Hong, W.P. Optical solitary wave solutions for the higher order nonlinear Schrodinger equation with cubic-quintic non-Kerr terms. Opt. Commun. 2001, 194, 217-223. [CrossRef]

13. Wazwaz, A.M.; El-Tantawy, S. Optical Gaussons for nonlinear logarithmic Schrodinger equations via the variational iteration method. Optik 2019, 180, 414-418. [CrossRef]

14. Kaur, L.; Wazwaz, A.M. Bright-dark optical solitons for Schrodinger-Hirota equation with variable coefficients. Optik 2019, 179, 479-484. [CrossRef]

15. Daftardar-Gejji, V.; Jafari, H. An iterative method for solving nonlinear functional equations. J. Math. Anal. Appl. 2006, 316, 753-763. [CrossRef]

16. Jafari, H. Iterative Methods for Solving System of Fractional Differential Equations. Ph.D. Thesis, Savitribai Phule Pune University, Pune, India, 2006.

17. Jafari, H.; Nazari, M.; Baleanu, D.; Khalique, C.M. A new approach for solving a system of fractional partial differential equations. Comput. Math. Appl. 2013, 66, 838-843. [CrossRef]

18. Yan, L. Numerical Solutions of Fractional Fokker-Planck Equations Using Iterative Laplace Transform Method. Abstr. Appl. Anal. 2013, 2013, 1-7. [CrossRef]

19. Prakash, A.; Kumar, M.; Baleanu, D. A new iterative technique for a fractional model of nonlinear Zakharov-Kuznetsov equations via Sumudu transform. Appl. Math. Comput. 2018, 334, 30-40. [CrossRef]

20. Ramadan, M.A.; Al-luhaibi, M.S. New iterative method for solving the fornberg-whitham equation and comparison with homotopy perturbation transform method. J. Adv. Math. Comput. Sci. 2014, 4, 1213-1227. [CrossRef]

21. Elzaki, T.M. The new integral transform Elzaki transform. Glob. J. Pure Appl. Math. 2011, 7, 57-64.

22. Elzaki, T.M. On the connections between Laplace and Elzaki transforms. Adv. Theor. Appl. Math. 2011, 6, 1-11.

23. Elzaki, T.M. On The New Integral Transform"Elzaki Transform"Fundamental Properties Investigations and Applications. Glob. J. Math. Sci. Theory Pract. 2012, 4, 1-13.

Publisher's Note: MDPI stays neutral with regard to jurisdictional claims in published maps and institutional affiliations.

(C) 2020 by the authors. Licensee MDPI, Basel, Switzerland. This article is an open access article distributed under the terms and conditions of the Creative Commons Attribution (CC BY) license (http:/ / creativecommons.org/licenses/by/4.0/). 